\title{
Discrete Time Option Pricing with Flexible Volatility Estimation
}

\author{
Wolfgang Härdle* Christian Hafner ${ }^{\dagger}$ \\ June 1997 \\ (first draft: October 1996)
}

\begin{abstract}
By extending the GARCH option pricing model of Duan (1995) to more flexible volatility estimation it is shown that the prices of out-of-the-money options strongly depend on volatility features such as asymmetry. Results are provided for the properties of the stationary pricing distribution in the case of a threshold GARCH model. For a stock index series with a pronounced leverage effect, simulated threshold GARCH option prices are substantially closer to observed market prices than the Black/Scholes and simulated GARCH prices.
\end{abstract}

\footnotetext{
*Institut für Statistik und Ökonometrie, Humboldt-Universität zu Berlin, Spandauer Str.1, D-10178 Berlin, Germany

${ }^{\dagger}$ CORE, Louvain-la-Neuve, Belgium, and SFB 373, Humboldt-Universität zu Berlin, Spandauer Str.1, D10178 Berlin, Germany; e-mail hafner@core.ucl.ac.be. This research was partially financed by contributions from the Deutsche Forschungsgemeinschaft, Sonderforschungsbereich 373 "Quantification and Simulation of Economic Processes" and by a research grant of the Belgian Government. Comments from Frédéric Jouneau, Eckhard Platen and Martin Schweizer are gratefully acknowledged.
} 


\section{Introduction}

It has long been recognized in the option pricing literature that the Black/Scholes prices reveal certain empirical anomalies, e.g. the well-known 'smile' effect. In recent years, the most prominent explanation for these anomalies has been stochastic volatility of the underlying asset. Empirically less significant are the effects of trading in discrete time (Bossaerts and Hillion, 1997) and feedback effects of hedging on the stock price process (Platen and Schweizer, 1997).

Since the introduction by Engle (1982), autoregressive conditional heteroskedasticity (ARCH) models have been successfully applied to financial time series. It is thus natural to consider pricing models for options on assets whose prices follow ARCH-type processes. To this end, Duan (1995) established a discrete-time option pricing model for the case of a GARCH volatility process. The aim of our paper is to show that for a given preference structure the results of Duan may be very sensitive to alternative specifications of the volatility process. This concerns the statistical properties of the asset price process under the equivalent martingale measure as well as the simulated prices.

The shape of the news impact curve, defined by Engle and $\mathrm{Ng}$ (1993) as today's volatility as a function of yesterday's return, is one of the dominating pricing factors. For instance, it is relevant to find out whether the news impact curve is symmetric or asymmetric, how fast it increases and whether it saturates for large returns. In general, far in- and far out-of-themoney options are underpriced and at-the-money options overpriced by Black/Scholes in the case of stochastic volatility. However, as the simulations of Hull and White (1987) already show, the degree of mispricing strongly depends on the volatility parameters and even more strongly on the correlation between volatility and the stock price.

In order to alleviate mispricing due to volatility misspecifation, flexible volatility models are required. If there is a correlation between stock price and volatility, one could use the EGARCH model of Nelson (1991). This model, however, has the drawback that stationarity conditions and the asymptotics of QMLE are not completely solved. An alternative way is to introduce thresholds for the news impact curve as in the threshold GARCH (TGARCH) model by Zakoian (1994, for the conditional standard deviation) and Glosten, Jagannathan and Runkle (1993, for the conditional variance). If the number of thresholds can be determined from the data this approach has the appealing property that it is the first step towards a nonparametric model without any parametric restriction. In fact, recent papers on nonparametric volatility estimation show that these models are able to reveal volatility features that would be difficult to capture with parametric models. Bossaerts, Härdle and Hafner (1996) obtain asymmetry of nonparametric news impact curves for major foreign exchange rates. Also, they show that the conditional kurtosis may not be constant, which is not consistent with the standard conditional normality assumption.

However, an exhaustive analysis of the complex structure of high frequency financial time series and its impact on option pricing has to be left to future research. Here, we focus solely 
on the volatility specification, knowing that the effects of e.g. skewness and kurtosis may not be negligible. We extend the results of Duan (1995) to the case of a TGARCH process and provide extensive Monte Carlo simulation results for three typical parameter constellations. In particular, we compare the simulated GARCH option prices with corresponding TGARCH and Black/Scholes prices. In an empirical analysis, we show that the observed call option market prices indeed reflect the asymmetry found for the news impact curve of a DAX series.

Section 2 gives a review of recent developments of volatility models in discrete time, Section 3 extends the GARCH option pricing model to TGARCH, Section 4 provides a simulation study for GARCH and TGARCH option prices and in Section 5 price predictions are obtained for calls on the DAX and compared with market prices.

\section{A succinct review of flexible ARCH models}

It is well-known that returns of financial time series exhibit nonconstant volatility patterns. A general time series model for financial returns would be

$$
y_{t}=\mu_{t}+\varepsilon_{t},
$$

with $\varepsilon_{t}=\sigma_{t} \xi_{t}, \quad \xi_{t} \sim$ i.i.d.(0,1), and $\mu_{t}$ and $\sigma_{t}$ being respectively the mean and standard deviation conditional on the past. $\sigma_{t}$ can be either stochastic itself or determined by the past history of the time series. If $\mu_{t}$ is interpreted as the risk premium, it can be linked to $\sigma_{t}$, as in the ARCH-in-mean (ARCH-M) model of Engle, Lilien, and Robins (1987). For $\mu_{t}=r+f\left(\sigma_{t}^{2}\right)$, $r$ would typically be the riskfree rate and $f$ the logarithm or square root.

The ARCH( $q$ ) model (Engle, 1982) assumes a linear dependence of the conditional variance on squared past residuals,

$$
\sigma_{t}^{2}=\omega+\sum_{i=1}^{q} \alpha_{i} \varepsilon_{t-i}^{2} .
$$

Bollerslev (1986) generalized the ARCH $(q)$ model to an analogue of ARMA processes for $\sigma_{t}^{2}$. The $\operatorname{GARCH}(p, q)$ model takes the form

$$
\sigma_{t}^{2}=\omega+\sum_{i=1}^{q} \alpha_{i} \varepsilon_{t-i}^{2}+\sum_{j=1}^{p} \beta_{j} \sigma_{t-j}^{2}
$$

The original idea of these ARCH type models of volatility was that the value of today's $\sigma_{t}$ is computable from recent innovation scale factors and/or past residuals of the process. The standard ARCH models have a defect though in the sense that they do not model possible asymmetric volatility shocks. "Good news" do not necessarily have the same impact on volatility as "bad news". Engle and Ng (1993) provide a survey of many parametric models proposed to overcome the symmetry problem. Important representatives in this context are the EGARCH model and the threshold ARCH models. 
Nelson (1991) introduced the exponential GARCH (EGARCH) model,

$$
\log \sigma_{t}^{2}=\omega_{t}+\sum_{k=1}^{\infty} \beta_{k} g\left(\xi_{t-k}\right)
$$

with deterministic coefficients $\omega_{t}, \beta_{k}$, and $g(u)=\gamma(|u|-E|u|)+\theta u$. The EGARCH model has several important advantages over the classic ARCH formulation of conditional heteroskedasticity. It models volatility more naturally in a multiplicative way, and the piecewise linear function $g$ may model the observable asymmetry of $\sigma_{t}^{2}$ as a function of past innovations. A disadvantage though is that for some common fat-tailed distributions of $\xi_{t}$ the unconditional variance is not finite. Also, it implies an exponential increase of the news impact curve, which has not been found favorable in many empirical investigations.

The idea of threshold ARCH (TARCH) models is to keep the functional form of the standard GARCH model, but to let the coefficients $\alpha$ depend on past innovations. Glosten, Jagannathan and Runkle (1993) consider the simple case where $\alpha$ depends only on the sign of the past innovation, i.e.

$$
\sigma_{t}^{2}=\omega+\alpha_{1} \varepsilon_{t-1}^{2} I\left(\varepsilon_{t-1}<0\right)+\alpha_{2} \varepsilon_{t-1}^{2} I\left(\varepsilon_{t-1}>0\right)+\beta \sigma_{t-1}^{2} .
$$

Here, $I(A)$ denotes an indicator function that takes the value one if the logical expression $A$ is true, and zero otherwise. Obviously, this model coincides with the standard GARCH $(1,1)$ model if $\alpha_{1}=\alpha_{2}$. The case $\alpha_{1}>\alpha_{2}$ describes the leverage effect, which is usually observed for stock returns.

In the same spirit, Zakoian (1994) modeled the conditional standard deviation. For one threshold at zero, a threshold ARCH model of order $q$ can be written as

$$
\sigma_{t}=\omega+\sum_{i=1}^{q} \alpha_{i}^{+} \varepsilon_{t-i}^{+}+\sum_{t=1}^{q} \alpha_{i}^{-} \varepsilon_{t-i}^{-},
$$

with $\varepsilon_{t}^{+}=\max \left(\varepsilon_{t}, 0\right)$ and $\varepsilon_{t}^{-}=\min \left(\varepsilon_{t}, 0\right)$. Rabemananjara and Zakoian (1993) applied this model in a generalized form to the French stock market. Recently, this model was generalized by El Babsiri and Zakoian (1996) by specifying $\varepsilon_{t}=\sigma_{t,+} \xi_{t}^{+}+\sigma_{t,-} \xi_{t}^{-}$, where $\sigma_{t,+}$ and $\sigma_{t,-}$ are TGARCH processes. Thus, depending on the sign of the innovations one possibly obtains different volatility processes.

A first step towards a flexible nonparametric modelling of volatility was made by the path-breaking paper of Gouriéroux and Monfort (1992). Their Qualitative Threshold ARCH (QTARCH) model had $\sigma_{t}^{2}$ as a step function of the past returns $y_{t}$. For instance, a QTARCH model of order one takes the form

$$
\sigma_{t}=\sum_{j=1}^{J} s_{j} I\left(y_{t-1} \in A_{j}\right),
$$

where $\left\{A_{j}\right\}_{j=1}^{J}$ is a partition of the real line, $s_{j}$ are the step heights and $J$ is the number of steps. 
A direct advantage of model (7) is that the functional form is no longer bound to a specific one, since step functions are dense in the $L_{2}$ function space. A disadvantage though is that the choice of $J$ is not flexible. Gouriéroux and Monfort (1992) assumed a known and fixed number of steps $J$.

A more flexible model is described in Härdle and Tsybakov (1997) where the volatility is modelled as an unknown function of the past return,

$$
\sigma_{t}^{2}=g\left(y_{t-1}\right)
$$

An extension to the multivariate case $\sigma_{t}^{2}=g\left(y_{t-1}, \ldots, y_{t-q}\right)$ is given by Härdle, Tsybakov and Yang (1997). In that paper a multivariate time series volatility matrix is modelled as an unknown function of the past values of the process. From the smoothing literature it is well known that the flexibility of free functional form estimation has to be paid with reduced statistical precision, especially in higher dimension. In the case considered here the consequence for the practical use of smoothing techniques for time series must be a limit on the number of lags or an introduction of lower dimensional structure.

The newer literature pursues the second way by considering additive models or multiplicative structures of volatility, see Härdle, Lütkepohl and Chen (1997), Yang and Härdle (1997) and Hafner (1997). Also, a nonparametric analogue of the heterogenous ARCH (HARCH) model of Müller et al. (1996) can be established as

$$
\sigma_{t}^{2}=\omega+\sum_{j=1}^{q} g_{j}\left(\sum_{i=1}^{j} y_{t-i}\right)
$$

where $g_{j}$ are nonparametric additive factor functions. This model is economically appealing, since it regards volatility as the accumulation of different market components. These components are described by the trader's frequency of regarding and acting at the market, each component having a different impact on volatility.

\section{Option pricing with alternative ARCH models}

We consider a discrete-time economy where interest rates and returns are paid after each time interval of fixed equispaced length. This contrasts the usual formulation in terms of continuously compounded interest rates and returns, but we keep the notation consistent with the notation traditionally used in the ARCH literature.

Let $S_{t}, t=0,1,2, \ldots$ be the price of a stock at time $t$ and $y_{t}=\left(S_{t}-S_{t-1}\right) / S_{t-1}$ be its oneperiod return excluding dividend payments. Suppose that there is a price for risk, measured in terms of a risk premium that is added to the riskfree rate $r$ to build the expected nextperiod return. It is sensible to allow dependence of risk premia on the conditional variance. As Duan (1995), we adopt the ARCH-M model of Engle, Lilien, and Robins (1987) with the risk 
premium being a linear function of the conditional standard deviation,

$$
\begin{aligned}
y_{t} & =r+\lambda \sigma_{t}+\varepsilon_{t} \\
\varepsilon_{t} \mid \mathcal{F}_{t-1} & \sim N\left(0, \sigma_{t}^{2}\right) \\
\sigma_{t}^{2} & =\omega+\alpha \varepsilon_{t-1}^{2}+\beta \sigma_{t-1}^{2} .
\end{aligned}
$$

In (12), $\omega, \alpha$, and $\beta$ are constant parameters satisfying stationarity and positivity conditions. The constant parameter $\lambda$ may be interpreted as the unit price for risk. $\mathcal{F}_{t}$ denotes the set of all information prior to and including time $t$. For notational convenience we restrict our discussion to the $\operatorname{GARCH}(1,1)$ case.

The above model is estimated under the empirical measure $P$. If one wants to apply the risk-neutral pricing methodology (see Cox and Ross, 1976), the measure has to be transformed such that the resulting discounted price process is a martingale. This guarantees that there are no arbitrage opportunities (Harrison and Kreps, 1979). Due to the incompleteness of markets, however, there is a multitude of such transformations (Harrison and Pliska, 1981). Unlike the complete market situation, incompleteness leaves the trader unable to construct a self-financing portfolio that exactly duplicates the option's payoff. Thus, hedging involves a risk, and option prices generally depend on risk preferences. It should be emphasized that the non-availability of a perfect hedging strategy is of eminent importance for the hedging practice.

To apply present value pricing by choosing a particular pricing measure, one has to impose assumptions about the pricing of volatility. Many papers investigated option prices under stochastic volatility for the case that volatility has zero systematic risk (i.e. the volatility risk premium is zero, see e.g. Hull and White, 1987, and Renault and Touzi, 1996). Melino and Turnbull (1990) allowed for nonzero, constant and exogenous volatility risk premia. As the empirical results of Wiggins (1987) show, the non-pricing of changes in volatility may not be justified.

Duan identified an equivalent martingale measure $Q$ by requiring that the conditional return distribution remains normal, and

$$
\operatorname{Var}^{P}\left(y_{t} \mid \mathcal{F}_{t-1}\right)=\operatorname{Var}^{Q}\left(y_{t} \mid \mathcal{F}_{t-1}\right)
$$

almost surely with respect to $P$. This is what he terms the 'locally risk-neutral valuation relationship' (LRNVR). He shows that a representative agent with, for example, constant relative risk aversion and normally distributed relative changes in aggregate consumption maximizes his expected utility using the LRNVR. The LRNVR incorporates a constant volatility risk premium that is directly linked to the risk premium in the mean. The alternative concept of minimizing the quadratic loss of a hedge portfolio, as pioneered by Föllmer and Sondermann (1986), will in general lead to a different choice of the pricing measure. The minimal equivalent martingale measure as defined by Föllmer and Schweizer (1991) is, intuitively, characterized by the smallest distance (in terms of a relative entropy, i.e. Kullback-Leibler distance) to the empirical measure, in the class of all equivalent martingale measures, $P^{*}$ say. It minimizes the 
expected quadratic difference between the option value and the value of a hedge portfolio at the terminal date. The following theorem states that under conditional normality (13) ensures minimization of the relative entropy of the conditional densities under $P$ and $P^{*}$, which we call local relative entropy. It is general in the sense that it does not depend on the specification of the volatility process $\sigma_{t}^{2}$.

Theorem 1 Given conditional normality under the class of equivalent martingale measures $P^{*}$, the LRNVR-measure minimizes the local relative entropy of $P^{*}$ with respect to $P$.

Proof: see Appendix.

Note that unlike the minimal martingale measure, the LRNVR measure is not concerned with the properties of the stationary distributions under $P$ and $P^{*}$, but with the conditional distributions. Both measures coincide if $y_{t}$ is conditionally homoskedastic, or if the unit risk premium $\lambda$ is zero.

To obtain a martingale process under the new measure, one has to introduce a new error term, $\eta_{t}$, that incorporates the time-varying risk premium effect. Hence, by defining $\eta_{t}=$ $\varepsilon_{t}+\lambda \sigma_{t}$, the LRNVR leads to the following model under the pricing measure $Q$ :

$$
\begin{aligned}
y_{t} & =r+\eta_{t} \\
\eta_{t} \mid \mathcal{F}_{t-1} & \sim N\left(0, \sigma_{t}^{2}\right) \\
\sigma_{t}^{2} & =\omega+\alpha\left(\eta_{t-1}-\lambda \sigma_{t-1}\right)^{2}+\beta \sigma_{t-1}^{2} .
\end{aligned}
$$

For the GARCH $(1,1)$ model, the variance of the stationary distribution under the empirical measure $P$ is $\operatorname{Var}^{P}\left(\varepsilon_{t}\right)=\omega /(1-\alpha-\beta)$, see Bollerslev (1986). For the LRNVR-measure the variance of the stationary distribution increases to $\operatorname{Var}^{Q}\left(\eta_{t}\right)=\omega /\left(1-\alpha\left(1+\lambda^{2}\right)-\beta\right)$ due to the fact that the volatility process under $Q$ is driven by noncentral rather than central chisquare distributed innovations. We will see below that the change of the unconditional variance crucially depends on the specification of the news impact curve.

As noted above, the restriction of having a quadratic and symmetric news impact function may not always be reasonable, as many empirical studies of stock returns showed. For the above model, this assumption can be relaxed to some nonlinear news impact function $g(\cdot)$. The following model is a nonparametric (or semiparametric) analogue to the GARCH model. Under the empirical measure $P$ we have

$$
\begin{aligned}
& y_{t}=r+\lambda \sigma_{t}+\varepsilon_{t} \\
& \varepsilon_{t} \mid \mathcal{F}_{t-1} \quad \sim_{P} \quad N\left(0, \sigma_{t}^{2}\right) \\
& \sigma_{t}^{2}=g\left(\varepsilon_{t-1}\right)+\beta \sigma_{t-1}^{2} \text {. }
\end{aligned}
$$

For this general framework with no prior information on $g(\cdot)$, estimation is a delicate issue, because iterative estimators are required. However, if $\beta$ is sufficiently small one can truncate at some lag and estimate a conventional semiparametric additive model. 
Under the LRNVR equivalent martingale measure $Q$, the model becomes

$$
\begin{aligned}
y_{t} & =r+\eta_{t} \\
\eta_{t} \mid \mathcal{F}_{t-1} & \sim_{Q} \\
\sigma_{t}^{2} & =g\left(0, \sigma_{t}^{2}\right)
\end{aligned}
$$

Note that once an estimate of $g(\cdot)$ is obtained under $P$, it can readily be used for the pricing under $Q$.

However, we decided not to use this general semiparametric model because a thorough analysis of the properties of the estimators is still in progress. Instead, we consider a flexible parametric model that will be investigated below in a simulation study, i.e. the threshold GARCH model of Glosten, Jagannathan and Runkle (1993), where the news impact function can be written as $g(x)=\omega+\alpha_{1} x^{2} I(x<0)+\alpha_{2} x^{2} I(x>0)$. To give some motivation for this model, we estimated a very simple nonparametric model, $y_{t}=\sigma\left(y_{t-1}\right) \xi_{t}$, for the returns on the German stock index DAX, which will be further analyzed in Section 5 . The estimate of the news impact curve $\sigma^{2}(\cdot)$ is shown in Figure 2. To have an idea about the distribution of the returns, a nonparametric density estimate vis-a-vis a smoothed normal density is provided in Figure 1.

$$
\text { Figures } 1 \text { and } 2
$$

It is obvious that $g(\cdot)$ is not symmetric around zero. Also, $g(\cdot)$ is not symmetric around a positive risk premium measure, since the average risk premium of the estimated TGARCH-M model in Section 5 is of smaller size. The TGARCH model captures this effect by having $\alpha_{1}>\alpha_{2}$. We are aware of the fact that other parametric models may as well describe this feature, but the TGARCH model has proven to be a sufficiently flexible and tractable model for stock returns (see, e.g., Rabemananjara and Zakoian, 1993), whereas the EGARCH model, as noted above, suffers from several theoretical and practical drawbacks.

Recall that the innovation distribution is normal. Thus, it follows for the TGARCH model that the unconditional variance under $P$ is $\operatorname{Var}^{P}\left(\varepsilon_{t}\right)=\omega /(1-\bar{\alpha}-\beta)$, with $\bar{\alpha}=\left(\alpha_{1}+\alpha_{2}\right) / 2$. The following theorem provides the unconditional variance under $Q$.

Theorem 2 The unconditional variance of the TGARCH(1,1) model under the LRNVR equivalent martingale measure $Q$ is

$$
\operatorname{Var}^{Q}\left(\eta_{t}\right)=\frac{\omega}{1-\psi(\lambda)\left(\alpha_{1}-\alpha_{2}\right)-\alpha_{2}\left(1+\lambda^{2}\right)-\beta}
$$

with

$$
\psi(u)=\frac{u}{\sqrt{2 \pi}} \exp \left(-\frac{1}{2} u^{2}\right)+\left(1+u^{2}\right) \Phi(u)
$$

and $\Phi(u)$ denoting the cumulative standard normal distribution function. 
Proof: see Appendix.

$\psi$ is a positive-valued function, and $\psi(\lambda)>1 / 2$ for the realistic case $\lambda>0$. We can make the following statements about the change of the unconditional variance: For (1) $\alpha_{1}=\alpha_{2}$, (17) coincides with the GARCH(1,1) result. For (2) $\alpha_{1}>\alpha_{2}$ (the leverage effect case), the unconditional variance increases even stronger than in the symmetric GARCH case. For (3) $\alpha_{1}<\alpha_{2}$, the unconditional variance will be smaller than for the leverage effect case, and we can distinguish two cases: If the inequality

$$
\alpha_{1}<\alpha_{2} \frac{2 \psi(\lambda)-1-2 \lambda^{2}}{2 \psi(\lambda)-1}
$$

holds, then the unconditional variance under $Q$ will be even smaller than the unconditional variance under $P$. If (18) does not hold, then we have as above $\operatorname{Var}^{P}\left(\varepsilon_{t}\right) \leq \operatorname{Var}^{Q}\left(\eta_{t}\right)$. However, the quotient on the right hand side of (18) takes negative values for realistic values of the unit risk premium (i.e. small positive values), such that for most empirical studies (18) will not hold.

Of course the stationary variance affects the option price: the larger (smaller) the variance, the higher (lower) the option price. This is especially relevant for long maturity options where the long run mean of volatility is one of the important determinants of the option price. Thus, options may be 'underpriced' when employing the GARCH model if in fact there is a leverage effect.

A second pecularity of the LRNVR approach is that under $Q$ and for positive risk premia, today's innovation is negatively correlated with tomorrow's GARCH conditional variance, contrary to the zero correlation under $P$. More precisely, we have $\operatorname{Cov}^{Q}\left(\eta_{t} / \sigma_{t}, \sigma_{t+1}^{2}\right)=$ $-2 \lambda \alpha \operatorname{Var}^{Q}\left(\eta_{t}\right)$ with GARCH parameter $\alpha$. This suggests that short run predictions of volatility under $Q$ (which affects the option price) depend not only on squared past innovations, but also on their signs. In particular, for $\lambda>0$ a negative (positive) past innovation tends to increase (decrease) volatility and thus the option price. The following theorem states that the covariance depends on the asymmetry of the news impact function when we use a TGARCH instead of a GARCH model.

Theorem 3 For the TGARCH(1,1) model, the covariance under the LRNVR equivalent martingale measure $Q$ of the innovation at time $t$ and the conditional variance at time $t+1$ can be expressed as

$$
\operatorname{Cov}^{Q}\left(\frac{\eta_{t}}{\sigma_{t}}, \sigma_{t+1}^{2}\right)=-2 \operatorname{Var}^{Q}\left(\eta_{t}\right)\left\{\lambda \alpha_{2}+\left[\frac{1}{\sqrt{2 \pi}} \exp \left(-\frac{1}{2} \lambda^{2}\right)+\lambda \Phi(\lambda)\right]\left(\alpha_{1}-\alpha_{2}\right)\right\},
$$

where $\operatorname{Var}^{Q}\left(\eta_{t}\right)$ is given in Theorem 2 .

Proof: see Appendix.

Assume in the following that we have a positive unit risk premium $\lambda$. Again, we can distinguish three cases: (1) For $\alpha_{1}=\alpha_{2}$ (the symmetry case), we obtain $\operatorname{Cov}^{Q}\left(\eta_{t} / \sigma_{t}, \sigma_{t+1}^{2}\right)=$ 
$-2 \lambda \alpha_{2} \operatorname{Var}^{Q}\left(\eta_{t}\right)$, i.e. the $\operatorname{GARCH}(1,1)$ result. For $\alpha_{1}<\alpha_{2}$ (the reverted leverage case) the covariance increases and if

$$
\lambda \alpha_{2}+\left[\frac{1}{\sqrt{2 \pi}} \exp \left(-\frac{1}{2} \lambda^{2}\right)+\lambda \Phi(\lambda)\right]\left(\alpha_{1}-\alpha_{2}\right)<0,
$$

then the correlation becomes positive. Finally, for $\alpha_{1}>\alpha_{2}$ (the leverage case) the covariance is negative and increases in absolute value.

This shows that also the reverting behavior of volatility to the stationary variance under $Q$ may be quite different from the symmetric GARCH case. The negative covariance is even larger for the leverage effect case. This indicates that options may be 'overpriced' ('underpriced') if the past innovation is positive (negative), the asset price follows a TGARCH process with $\alpha_{1}>\alpha_{2}$, and the GARCH model $\left(\alpha_{1}=\alpha_{2}\right)$ is used for volatility predictions.

\section{A Monte Carlo study}

Because the discounted price process is a martingale under the equivalent martingale measure $Q$, we can apply the risk-neutral valuation methodology of Cox and Ross (1976). The $Q-$ price of a call option at time $t, C_{t}$, is given by

$$
C_{t}=(1+r)^{-n} \mathrm{E}^{Q}\left[\max \left(S_{T}-K, 0\right) \mid \mathcal{F}_{t}\right]
$$

with $T$ denoting the maturity date, $n \equiv T-t$ the time to maturity and $K$ the exercise price. For European options, the no-arbitrage price of a put option, $P_{t}$, is determined by putcall parity, i.e., $P_{t}=C_{t}-S_{t}+(1+r)^{-n} K$. Because there is no analytic expression for the expectation in (21), we have to use numerical techniques to simulate the option price. That is, the distribution of the payoff function $\max \left(S_{T}-K, 0\right)$ at the terminal date is simulated by generating $m$ independent realizations of the stock price process

$$
S_{T, i}=S_{t} \prod_{s=t+1}^{T}\left(1+y_{s, i}\right), \quad i=1, \ldots, m,
$$

where $y_{s, i}$ is the return of the $i^{t h}$ realization at time $s$, and then discount the mean of the payoff-function with the riskfree rate, i.e.

$$
C_{t}=(1+r)^{-n} \frac{1}{m} \sum_{i=1}^{m} \max \left(S_{T, i}-K, 0\right) .
$$

Throughout the simulation study we used the following parameters: $r=0, S_{0}=100, n=30$ days, $m=400,000, \lambda=0.01$. The moneyness $S_{0} / K$ was varied from 0.85 to 1.15 , which is the typical range of traded options at the market. We do not compare the effects of different times to maturity $n$, because it is known from previous work that many of the features caused by stochastic volatility such as smiles disappear when the time to maturity is increased. In 


\begin{tabular}{ccccc}
\hline \hline Type & $\alpha$ & $\beta$ & $\alpha+\beta$ & $\rho_{1}$ \\
\hline 1 & 0.1 & 0.85 & 0.95 & 0.1791 \\
2 & 0.5 & 0.45 & 0.95 & 0.8237 \\
3 & 0.1 & 0.5 & 0.6 & 0.1077 \\
\hline \hline
\end{tabular}

Table 1: Characterization of types

general, these effects qualitatively stay the same, but quantitatively become more and more insignificant. This was confirmed by our experiments, so we focused on only one short maturity.

To reduce the variance of the payoffs, the antithetic variable technique of Hammersley and Handscomb (1964) was used. This turned out to be sufficient, since the standard errors of the obtained option prices were small due to our large number of replications $m$.

In order to study the effects of an asymmetric news impact function on option prices, we consider three situations, characterized by the degree of short-run autocorrelation of squared returns and by the degree of persistence. For a $\operatorname{GARCH}(1,1)$ process it can be shown that the first order autocorrelation of squared returns, $\rho_{1}$, is given by

$$
\rho_{1}=\alpha\left(1-\alpha \beta-\beta^{2}\right) /\left(1-2 \alpha \beta-\beta^{2}\right)
$$

and $\rho_{j}=(\alpha+\beta) \rho_{j-1}, j=2,3, \ldots$ Table 1 reports the parameter constellations and characteristics of the three types.

Type 1 is described by high persistence and small first order autocorrelation, Type 2 by high persistence and large first order autocorrelation, and Type 3 by low persistence and small first order autocorrelation. Type 1 is typical for high-frequency financial series (daily or intradaily), because it is usually observed that the autocorrelation function of squared returns drops quickly for the first lags but then declines very slowly. Type 2 describes a situation where there are very strong ARCH effects, and Type 3 resembles the case of highly aggregated data, e.g. monthly or quarterly series. In all cases the parameter $\omega$ is chosen such that $\sigma^{2}=0.0002$, i.e. the unconditional variance remains the same.

Concerning the nonlinear news impact function $g(\cdot)$, we have chosen the Threshold ARCH Model of Glosten, Jagannathan and Runkle (1993) and Zakoian (1994) with two asymmetry cases: The first case, which we may call 'leverage-effect' case, is

$$
g_{1}(x)=\omega+1.2 \alpha x^{2} I(x<0)+0.8 \alpha x^{2} I(x \geq 0)
$$

and the second, 'reverted leverage-effect' case

$$
g_{2}(x)=\omega+0.8 \alpha x^{2} I(x<0)+1.2 \alpha x^{2} I(x \geq 0) .
$$

The simulation results are summarized as follows: For each type, we generated plots of the absolute and relative difference of GARCH/TGARCH prices to the corresponding Black/Scholes 
price. The relative difference is defined as the absolute difference divided by the Black/Scholes price. Due to the small grid (we used steps of 0.01 for the moneyness), the functions appear very smooth. Additional to the plots we present the numerical results for selected values of the moneyness.

The results for the leverage effect case and Type 1 are summarized by Figure 3 and Table 5.

Figure 3 and Table 5

For the GARCH case we obtain the well-known result that the price difference to Black/Scholes has a U-shape with respect to the moneyness. As a consequence of the monotonously increasing call price in the moneyness, the relative difference is largest in absolute value for out-of-themoney options, whereas the relative difference becomes more and more negligible the higher the moneyness. This may also explain the often observed skewness of the smile effect. For the TGARCH option prices we basically observe a similar deviation to Black/Scholes but with one major difference: For the leverage effect, out-of-the-money options are priced lower and in-themoney-options higher than under a GARCH model. This is intuitively plausible: If an option is far out-of-the-money and time to maturity is short, the only possibility to be of positive value at the expiration date is that the underlying stock appreciates several times in a row with large returns. This, however, is less probable for the leverage case, because positive returns have in this case a smaller impact on volatility than in the symmetric case, provided that the above parameter constellation holds.

For the leverage effect case, the described deviation of TGARCH prices from GARCH prices is also visible for the other types (see Figures 5, 7 and Tables 6, 7).

Figures 5, 7 and Tables 6,7

For the reverted leverage effect case the arguments are reversed. Now it is more probable that an out-of-the-money option will end up in the money, and therefore the TGARCH prices of far out-of-the-money options are higher than the GARCH prices. The results for the reverted leverage case are given in Figures 4, 6 and 8 and Tables 8, 9 and 10.

Figures 4, 6, 8 and Tables 8, 9 and 10 


\begin{tabular}{cccccccc}
\hline \hline & $\omega$ & $\alpha$ & $\alpha_{1}$ & $\alpha_{2}$ & $\beta$ & $\lambda$ & $-2 \log L$ \\
\hline GARCH & $1.66 \mathrm{E}-05$ & 0.1438 & & & 0.7756 & 0.0691 & $-7,697.66$ \\
& $(1.04 \mathrm{E}-06)$ & $(0.0061)$ & & & $(0.012)$ & $(0.0178)$ & \\
\hline TGARCH & $1.91 \mathrm{E}-05$ & & 0.2005 & 0.0454 & 0.7736 & 0.0385 & $-7,719.24$ \\
& $(1.359 \mathrm{E}-06)$ & & $(0.0084)$ & $(0.0113)$ & $(0.0157)$ & $(0.0175)$ & \\
\hline \hline
\end{tabular}

Table 2: GARCH and TGARCH estimation results for DAX returns, 88/01/01-91/12/30 (standard errors in parentheses)

As one might expect, the deviations of the simulated prices to Black/Scholes and between the GARCH and TGARCH prices are highest for Type 2, i.e. for very strong short-run ARCH effects, and smallest for the low persistence Type 3. The latter case is expected, because the differences should disappear the more the homoskedastic case is approached.

\section{Application to the pricing of DAX Calls}

The GARCH pricing methodology was applied to German stock index and option data. As a stock index we used the daily closing notation of the DAX, January 1st, 1988 to April 30th, 1993. The closing notation of this index is usually fixed at about 13:30 local time (Frankfurt). For call options on this index we used the tick price recording of the DTB for January 1992. In order to synchronize stock and option time, we linearly interpolated between the last option price before 13:30 and the first one after, unless there was more than two hours difference.

There was no evidence for autocorrelation in the mean, but squared and absolute returns were highly autocorrelated, so the GARCH(1,1)-M model

$$
\begin{aligned}
y_{t} & =\lambda \sigma_{t}+\varepsilon_{t} \\
\varepsilon_{t} \mid \mathcal{F}_{t-1} & \sim N\left(0, \sigma_{t}^{2}\right) \\
\sigma_{t}^{2} & =\omega+\alpha \varepsilon_{t-1}^{2}+\beta \sigma_{t-1}^{2}
\end{aligned}
$$

was estimated by Quasi Maximum Likelihood Estimation for the DAX series until 1991/12/30. A constant parameter in (25) was not significant, so excluded from the estimation. The results are reported in Table 2.

All parameters are significant. The volatility is stationary with $\alpha+\beta=0.9194$. The risk premium parameter, $\lambda$, is positive as expected.

The QMLE results for the TGARCH model

$$
\sigma_{t}^{2}=\omega+\alpha_{1} \varepsilon_{t-1}^{2} I\left(\varepsilon_{t-1}<0\right)+\alpha_{2} \varepsilon_{t-1}^{2} I\left(\varepsilon_{t-1}>0\right)+\beta \sigma_{t-1}^{2}
$$

are also given in Table 2. 
Considering the log-Likelihood value, the TGARCH fit improves the GARCH fit. A likelihood-ratio test rejects the GARCH model at all conventional levels. $\alpha_{1}$ and $\alpha_{2}$ are significantly different, and thus the asymmetry of the news impact function is significant. Because $\alpha_{1}>\alpha_{2}$, we have the usual leverage effect for stock market series.

Having estimated the parameters, the next step is to simulate option prices for the subsequent period. We selected a call option, and since the DAX options traded at the DTB are of the European type, results for put options can be obtained by put-call parity. Also, in the light of the discussion above, a short maturity option was selected to capture those options that are most sensitive to stochastic volatility. The last trading day of the DAX call January was Friday, January 17. Three exercise prices $K$ were selected: 1550, 1600, and 1650, approximately reflecting the in-the-money, at-the-money and out-of-the-money cases, respectively. We simulated option prices for the ten days from January 3 to January 16.

One major problem is how to specify the starting value for the volatility process. A somewhat rash choice would be, as one usually does when estimating a model, to set it equal to the unconditional variance. As this is a good choice for an in-sample situation, it fails when it comes to out-of-sample prediction, because the current state is relevant. Therefore we experimented first setting the starting values equal to the current estimates of volatility by extrapolating the volatility process, keeping the parameters fixed. However, it became obvious that this procedure still gave a poor estimate of what the market's perception of 'risk' was at that time. In fact, it was seen that the implied volatility $\sigma_{i m}$ was substantially smaller than the GARCH and TGARCH estimates. For a given $r$, the implied volatility is defined by

$$
C_{\text {Market }}\left(S_{t}, K, n\right) \equiv C_{B S}\left(S_{t}, K, n, \sigma_{i m}\left(S_{t}, K, n\right)\right)
$$

where $C_{B S}$ is the option price in the standard model of Black and Scholes (1973), i.e.

$$
C_{B S}(S, K, n, \sigma)=S \Phi\left(d_{1}\right)-K e^{-r n} \Phi\left(d_{2}\right),
$$

with

$$
d_{1}=\frac{\ln (S / K)+\left(r+\sigma^{2} / 2\right) n}{\sigma \sqrt{n}}
$$

and $d_{2}=d_{1}-\sigma \sqrt{n}$.

We saw this large discrepancy between implied and estimated volatility as an indicator that the market had incremental information about the volatility state and, possibly, about future events. Thus, for the simulation of option prices at time $t$ we made use of the implied volatility $\sigma_{i m}\left(S_{t-1}, K, n+1\right)$ in two ways: First, as starting value for the (T)GARCH simulations, and second, as the markets expectation of the average volatility until maturity, i.e. $\omega$ is adjusted to

$$
\omega \equiv\left[1-\left(1+\lambda^{2}\right) \alpha-\beta\right] \sigma_{i m}^{2}\left(S_{t-1}, K, n+1\right)
$$

for the GARCH case, and

$$
\omega \equiv\left[1-\psi(\lambda)\left(\alpha_{1}-\alpha_{2}\right)-\left(1+\lambda^{2}\right) \alpha_{2}-\beta\right] \sigma_{i m}^{2}\left(S_{t-1}, K, n+1\right)
$$


for the TGARCH case with the function $\psi$ as defined in Theorem 2. Apart from the volatility, the other parameters are fixed at their estimated values.

For calculation of the Black/Scholes prices at time $t$, the implied volatility at time $t-1$ was used. A similar procedure was used in Bossaerts and Hillion (1993), where 15 minute old implied volatilities were plugged into the Black/Scholes formula which then performed well.

The prediction results and comparison with the actual market prices are reported in Table 3. At first glance the results appear mixed. It is striking that for some days (e.g. 01/14) the predicted option prices are far away from market prices. The only possible explanation is that the market's perception of risk, mirrored by the implied volatility, changed dramatically over one day. Because the implied volatility from the previous day is a crucial parameter for the above described prediction procedure, all three models fail in this case. Considering all ten days, however, the employed procedure was still doing much better than the procedure with current volatility estimates as starting values.

In order to have a goodness-of-fit criterion, we define relative residuals as

$$
u_{i, t} \equiv \frac{C_{i, t}-C_{\text {Market }, t}}{C_{\text {Market }, t}}
$$

with $i=$ BS,GARCH,TGARCH. Residuals should be looked at in relative terms, because traders will always prefer a cheap option which is 'underpriced' by the same amount as an expensive option, simply by multiplying his position in the cheap option. A similar argument applies for the case of selling 'overpriced' options. Due to the symmetry we can consider a quadratic loss-criterion, i.e.

$$
Q_{i}=\sum_{t} u_{i, t}^{2}
$$

The results for the three models are given in Table 4 .

Overall, both the GARCH and TGARCH perform substantially better than the Black/Scholes model. For in-the-money and at-the-money options, the improvement of the TGARCH prediction over GARCH is small. For out-of-the-money options, however, there is a large reduction of the loss criterion. Recall from the simulation study that options reacting most sensitive to stochastic volatility and leverage effects are out-of-the-money options. Now we obtain the same result for real market data, which tells us that the market is aware (or, at least, reflects it in the prices) of the asymmetry of the volatility. Thus, the conclusion is that it strongly matters which volatility process is estimated and used for option pricing.

\section{Conclusions}

In this paper, we show that out-of-the-money options strongly depend on the volatility specification. In particular, if there is a leverage effect, out-of-the-money options may be severely overpriced by assuming a symmetric news impact function, as in the GARCH model. For this to show, a simulation study was performed which used as the volatility generating process 


\begin{tabular}{|l|rrrrr|}
\hline \hline Date & $\mathrm{K}$ & $\mathrm{BS}$ & GARCH & TGARCH & Market \\
\hline \multirow{3}{*}{$92 / 01 / 03$} & 1550 & 62.3319 & 60.1093 & 60.6431 & 59.67 \\
& 1600 & 21.4640 & 20.6517 & 21.1346 & 20.75 \\
& 1650 & 3.4582 & 3.7396 & 3.5022 & 3.50 \\
\hline \multirow{3}{*}{$92 / 01 / 06$} & 1550 & 58.6185 & 57.6123 & 58.1194 & 57.50 \\
& 1600 & 19.6230 & 18.5490 & 18.9011 & 18.50 \\
& 1650 & 2.9354 & 2.8022 & 2.4888 & 2.00 \\
\hline & 1550 & 47.7014 & 46.3814 & 46.6255 & 49.33 \\
$92 / 01 / 07$ & 1600 & 12.2857 & 11.0794 & 11.1795 & 12.00 \\
& 1650 & 0.8013 & 0.8870 & 0.6543 & 1.00 \\
\hline & 1550 & 37.8624 & 35.7387 & 36.2037 & 35.39 \\
$92 / 01 / 08$ & 1600 & 6.2125 & 5.4397 & 5.3827 & 5.00 \\
& 1650 & 0.2602 & 0.3245 & 0.2043 & 1.00 \\
\hline \multirow{3}{*}{$92 / 01 / 09$} & 1550 & 43.2450 & 42.3468 & 42.5976 & 44.81 \\
& 1600 & 7.5984 & 6.8580 & 7.0327 & 6.70 \\
& 1650 & 1.2997 & 0.9276 & 0.6857 & 0.53 \\
\hline & 1550 & 66.8592 & 66.4068 & 66.6565 & 64.40 \\
$92 / 01 / 10$ & 1600 & 19.9157 & 19.3750 & 19.8060 & 19.50 \\
& 1650 & 2.1863 & 1.6141 & 1.4251 & 1.10 \\
\hline & 1550 & 72.6700 & 72.6602 & 72.6163 & 71.40 \\
$92 / 01 / 16$ & 1600 & 66.3400 & 66.3400 & 66.3400 & 67.35 \\
& 1650 & 16.3687 & 16.3400 & 16.3400 & 17.9 \\
\hline \hline $92 / 01 / 13$ & 1600 & 24.3434 & 23.6230 & 23.8160 & 24.50 \\
& 1650 & 1.3425 & 0.8010 & 0.6673 & 1.30 \\
\hline & 1550 & 78.5300 & 78.5290 & 78.5850 & 73.26 \\
& 1600 & 29.1454 & 28.7760 & 28.8189 & 26.00 \\
& 1650 & 1.4143 & 0.6909 & 0.6427 & 0.90 \\
\hline & 1550 & 117.5000 & 117.4606 & 117.4840 & 115.00 \\
& 1600 & 67.5000 & 67.4923 & 67.5102 & 65.00 \\
& 1650 & 18.2700 & 17.6215 & 17.6941 & 17.00 \\
\hline & 1550 & 116.3400 & 116.3400 & 116.3400 & 116.13 \\
& & & & \\
\hline
\end{tabular}

Table 3: Day-to-day price predictions for DAX calls traded during the period 1992/01/03 and 1992/01/16, maturing at 1992/01/17. BS: Black/Scholes price with volatility set to the implied volatility at the previous trading day. GARCH/TGARCH: Simulated prices with volatility process set to the estimated GARCH respectively TGARCH models. The estimation period was 1988/01/01 - 1991/12/30. 


\begin{tabular}{|c|ccc|}
\hline \hline K & BS & GARCH & TGARCH \\
\hline 1550 & 0.0170 & 0.0137 & 0.0136 \\
1600 & 0.0991 & 0.0286 & 0.0283 \\
1650 & 4.2305 & 1.6261 & 1.3139 \\
\hline total & 4.3465 & 1.6684 & 1.3558 \\
\hline \hline
\end{tabular}

Table 4: $Q_{i}$ for DAX calls traded during the period 1992/01/03 - 1992/01/16 and maturing at $1992 / 01 / 17$.

a constant (Black/Scholes), GARCH and Threshold GARCH. The TGARCH option prices of about more than five percent out-of-the-money options significantly deviated from the GARCH prices. In a real data example, it was shown for calls on the German stock index DAX maturing January 1992 that the simulated TGARCH prices were closer to market prices than both Black/Scholes and GARCH prices. In fact, under time-varying volatility and short maturity Black/Scholes seems to perform quite poorly, whereas GARCH and TGARCH both do reasonably well. The difference between GARCH and TGARCH becomes obvious when looking at the prices for options with high exercise price. Concluding, it can be stated that at least for the examined period in January 1992 traders at the DTB were aware of both the underlying stochastic volatility and the underlying leverage effect. The observed market prices reflect both of these features.

Future research will have to investigate the performance of standard hedge portfolios under different choices of the martingale measure as well as under misspecification of the time series model for the underlying stock.

\section{References}

Black, F., Scholes, M. (1973) The Pricing of Options and Corporate Liabilities, Journal of Political Economy 81: 637-659.

Bollerslev, T. (1986) Generalized Autoregressive Conditional Heteroskedasticity, Journal of Econometrics 31: 307-327.

Bossaerts, P., Härdle, W., Hafner, C. (1996) Foreign Exchange Rates Have Surprising Volatility, in: P.M.Robinson (ed.), Athens Conference on Applied Probability and Time Series, vol.2, Lecture Notes in Statistics 115, 55-72, Springer Verlag.

Bossaerts, P., Hillion, P. (1993) A Test of a General Equilibrium Stock Option Pricing Model, Mathematical Finance 3:311-347. 
Bossaerts, P., Hillion, P. (1997) Local Parametric Analysis of Hedging in Discrete Time, forthcoming in Journal of Econometrics.

Cox, J.C., Ross, S.A. (1976) The Valuation of Options for Alternative Stochastic Processes, Journal of Financial Economics 3: 145-166.

Duan, J.-C. (1995) The GARCH option pricing model, Mathematical Finance 5: 13-32.

El Babsiri, M., Zakoian, J.-M. (1996) Contemporaneous Asymmetry in Weak GARCH Processes, CORE DP 9604.

Engle, R. (1982) Autoregressive Conditional Heteroskedasticity with Estimates of the Variance of U.K. inflation, Econometrica 50: 987-1008.

Engle, R., Lilien, D., Robins, R. (1987) Estimating Time Varying Risk Premia in the Term Structure: The ARCH-M Model, Econometrica 55: 391-407.

Engle, R., Ng, V. (1993) Measuring and Testing the Impact of News on Volatility, Journal of Finance, 48: 1749-1778.

Föllmer, H., Schweizer, M. (1991) Hedging of Contingent Claims under Incomplete Information, in: Applied Stochastic Analysis, ed. by M.H.A.Davis and R.J.Elliot, Gordon and Breach, London 1991.

Föllmer, H., Sondermann, D. (1986) Hedging of Non-redundant Contingent Claims, in: Hildenbrand, W., Mas-Colell, A. (eds.), Contributions to Mathematical Economics, Amsterdam, North Holland, 205-223.

Glosten, L.R., Jagannathan, R., Runkle, D.E. (1993) On the Relation Between the Expected Value and the Volatility of the Nominal Excess Return on Stocks, Journal of Finance 48, 1779-1801.

Gouriéroux, C., Monfort, A. (1992) Qualitative Threshold ARCH Models, Journal of Econometrics 52: 159-199.

Härdle, W., Lütkepohl, H., Chen, R. (1997) A Review of Nonparametric Time Series Analysis, forthcoming in the International Statistical Review.

Härdle, W., Tsybakov, A. Local Polynomial Estimation of the Volatility Function, Journal of Econometrics.

Härdle, W., Tsybakov, A., Yang, L. (1996) Nonparametric Vector Autoregression, Journal of Statistical Planning and Inference.

Hafner, C. (1997) Estimating High Frequency Foreign Exchange Rate Volatility with Nonparametric ARCH Models, forthcoming in Journal of Statistical Planning and Inference. 
Hammersley, J.M., Handscomb, D.C. (1964) Monte Carlo Methods, Methuen, London.

Harrison, M., Kreps, D. (1979) Martingales and Arbitrage in Multiperiod Securities Markets, Journal of Economic Theory 20: 381-408.

Harrison, M., Pliska, S. (1981) Martingales and Stochastic Integrals in the Theory of Continuous Trading, Stochastic Processes Applications 11: 215-260.

Hull, J., White, A. (1987) The Pricing of Options on Assets with Stochastic Volatilities, Journal of Finance.

Melino, A., Turnbull, S.M. (1990) Pricing Foreign Currency Options with Stochastic Volatility, Journal of Econometrics 45: 239-265.

Müller, U.A., Dacorogna, M.M., Olsen, R.B., Pictet, O.V., von Weizsäcker, J.E. (1996) Volatilities of Different Time Resolutions - Analyzing the Dynamics of Market Components, Olsen \& Associates Discussion Paper.

Nelson, D. (1991) Conditional Heteroskedasticity in Asset Returns: A New Approach, Econometrica 59: $347-370$.

Platen, E., Schweizer, M. (1997) On Feedback Effects From Hedging Derivatives, forthcoming in Mathematical Finance.

Rabemananjara, R., Zakoian, J.M. (1993) Threshold ARCH Models and Asymmetries in Volatility, Journal of Applied Econometrics 8: 31-49.

Renault, E., Touzi, N. (1996) Option Hedging and Implied Volatilities in a Stochastic Volatility Model, Mathematical Finance.

Wiggins, J. (1987) Option Values under Stochastic Volatility: Theory and Empirical Estimates, Journal of Financial Economics 19: 351-372.

Yang, L., Härdle, W. (1997) Nonparametric Autoregression with Multiplicative Volatility and Additive Mean, forthcoming in Journal of Time Series Analysis.

Zakoian, J.M. (1994) Threshold Heteroskedastic Functions, Journal of Economic Dynamics and Control 18: 931-955. 


\section{Appendix}

Proof of Theorem 1. Let $f_{P^{*}}$ be the conditional distribution of $y_{t}$ under the equivalent martingale measure $P^{*}$. In the GARCH option pricing model, $f_{P^{*}}$ is normal with mean $r$ and variance, say, $v_{t}^{2}$. The conditional distribution under $P, f_{P}$, is also normal with mean $r+\lambda \sigma_{t}$ and variance $\sigma_{t}^{2}$. Let $\eta_{t} \mid \mathcal{F}_{t-1} \sim N\left(0, \sigma_{t}^{2}\right)$ under $P^{*}$. To determine the $v_{t}^{2}$ that minimizes the relative entropy (or Kullback-Leibler discrepancy) $d\left(P^{*} \mid P\right)$, we write

$$
\begin{aligned}
d\left(P^{*} \mid P\right) & =\int\left\{\log \frac{f_{P^{*}}(x)}{f_{P}(x)}\right\} f_{P^{*}}(x) d x \\
& =\int\left\{\log \frac{\sigma_{t}}{v_{t}}-\frac{1}{2 v_{t}^{2}}(x-r)^{2}+\frac{1}{2 \sigma_{t}^{2}}\left(x-\lambda \sigma_{t}-r\right)^{2}\right\} f_{P^{*}}(x) d x \\
& =\log \frac{\sigma_{t}}{v_{t}}-\frac{1}{2 v_{t}^{2}} \mathrm{E}^{P^{*}}\left[\eta_{t}^{2} \mid \mathcal{F}_{t}\right]+\frac{1}{2 \sigma_{t}^{2}} \mathrm{E}^{P^{*}}\left[\left(\eta_{t}-\lambda \sigma_{t}\right)^{2} \mid \mathcal{F}_{t}\right] \\
& =\log \frac{\sigma_{t}}{v_{t}}-\frac{1}{2 v_{t}^{2}} v_{t}^{2}+\frac{1}{2 \sigma_{t}^{2}} \mathrm{E}^{P^{*}}\left[\eta_{t}^{2}-2 \lambda \sigma_{t} \eta_{t}+\lambda^{2} \sigma_{t}^{2} \mid \mathcal{F}_{t}\right] \\
& =\log \frac{\sigma_{t}}{v_{t}}-\frac{1}{2}+\frac{1}{2 \sigma_{t}^{2}}\left(v_{t}^{2}+\lambda^{2} \sigma_{t}^{2}\right) \\
& =\log \frac{\sigma_{t}}{v_{t}}-\frac{1}{2}+\frac{v_{t}^{2}}{2 \sigma_{t}^{2}}+\frac{\lambda^{2}}{2}
\end{aligned}
$$

The first order condition for a minimum of $d\left(P^{*} \mid P\right)$ with respect to $v_{t}$ is

$$
-\frac{1}{v_{t}}+\frac{v_{t}}{\sigma_{t}^{2}}=0
$$

This gives

$$
v_{t}^{2}=\sigma_{t}^{2},
$$

and since the second order condition for a minimum is satisfied, we have

$$
Q=\arg \min _{P^{*}} d\left(P^{*} \mid P\right),
$$

and the proof is complete. Q.E.D.

Proof of Theorem D. Let $z_{t}=\eta_{t} / \sigma_{t}-\lambda$. Under $Q, z_{t} \mid \mathcal{F}_{t-1} \sim N(-\lambda, 1)$. The conditional variance can be written as

$$
\sigma_{t}^{2}=\omega+\alpha_{1} \sigma_{t-1}^{2} z_{t-1}^{2} I\left(z_{t-1}<0\right)+\alpha_{2} \sigma_{t-1}^{2} z_{t-1}^{2} I\left(z_{t-1}>0\right)+\beta \sigma_{t-1}^{2} .
$$

Taking expectations, the integral expression for the negative support can be verified to be

$$
\begin{aligned}
\mathrm{E}^{Q}\left[z_{t}^{2} I\left(z_{t}<0\right) \mid \mathcal{F}_{t-1}\right] & =\frac{1}{\sqrt{2 \pi}} \int_{-\infty}^{0} z^{2} e^{-\frac{1}{2}(z+\lambda)^{2}} d z \\
& =\frac{1}{\sqrt{2 \pi}} \int_{-\infty}^{\lambda}(u-\lambda)^{2} e^{-\frac{1}{2} u^{2}} d u \\
& =\frac{\lambda}{\sqrt{2 \pi}} e^{-\frac{1}{2} \lambda^{2}}+\left(1+\lambda^{2}\right) \Phi(\lambda) \\
& =: \psi(\lambda) .
\end{aligned}
$$


Since

$$
\mathrm{E}^{Q}\left[z_{t}^{2} \mid \mathcal{F}_{t-1}\right]=\frac{1}{\sqrt{2 \pi}} \int_{-\infty}^{\infty} z^{2} e^{-\frac{1}{2}(z+\lambda)^{2}} d z=1+\lambda^{2},
$$

we have for the positive support

$$
\mathrm{E}^{Q}\left[z_{t}^{2} I\left(z_{t}>0\right) \mid \mathcal{F}_{t-1}\right]=1+\lambda^{2}-\psi(\lambda)
$$

Thus, we arrive at

$$
\mathrm{E}^{Q}\left[\sigma_{t}^{2}\right]=\omega+\alpha_{1} \psi(\lambda) \mathrm{E}^{Q}\left[\sigma_{t-1}^{2}\right]+\alpha_{2}\left[1+\lambda^{2}-\psi(\lambda)\right] \mathrm{E}^{Q}\left[\sigma_{t-1}^{2}\right]+\beta \mathrm{E}^{Q}\left[\sigma_{t-1}^{2}\right]
$$

Noting that the unconditional variance is independent of $t$, the result is obtained. Q.E.D.

Proof of Theorem 3. At first, the conditional covariance is determined:

$$
\begin{aligned}
\operatorname{Cov}_{t-1}^{Q}\left(\frac{\eta_{t}}{\sigma_{t}}, \sigma_{t+1}^{2}\right) & =\alpha_{1} \mathrm{E}_{t-1}^{Q}\left[\frac{\eta_{t}}{\sigma_{t}}\left(\eta_{t}-\lambda \sigma_{t}\right)^{2} I\left(\eta_{t}-\lambda \sigma_{t}<0\right)\right]+ \\
& +\alpha_{2} \mathrm{E}_{t-1}^{Q}\left[\frac{\eta_{t}}{\sigma_{t}}\left(\eta_{t}-\lambda \sigma_{t}\right)^{2} I\left(\eta_{t}-\lambda \sigma_{t}>0\right)\right]
\end{aligned}
$$

where $\mathrm{E}_{t}(\cdot)$ and $\operatorname{Cov}_{t}(\cdot)$ abbreviates $\mathrm{E}\left(\cdot \mid \mathcal{F}_{t}\right)$ and $\operatorname{Cov}\left(\cdot \mid \mathcal{F}_{t}\right)$, respectively. The first conditional expectation on the right hand side of (38) can be shown to be

$$
\mathrm{E}_{t-1}^{Q}\left[\frac{\eta_{t}}{\sigma_{t}}\left(\eta_{t}-\lambda \sigma_{t}\right)^{2} I\left(\eta_{t}-\lambda \sigma_{t}<0\right)\right]=-2 \sigma_{t}^{2}\left[\frac{1}{\sqrt{2 \pi}} \exp \left(-\frac{1}{2} \lambda^{2}\right)+\lambda \Phi(\lambda)\right] .
$$

Because of $\mathrm{E}_{t-1}^{Q}\left[\frac{\eta_{t}}{\sigma_{t}}\left(\eta_{t}-\lambda \sigma_{t}\right)^{2}\right]=-2 \lambda \sigma_{t}^{2}$, we can write for the second conditional expectation in $(38)$

$$
\mathrm{E}_{t-1}^{Q}\left[\frac{\eta_{t}}{\sigma_{t}}\left(\eta_{t}-\lambda \sigma_{t}\right)^{2} I\left(\eta_{t}-\lambda \sigma_{t}>0\right)\right]=-2 \sigma_{t}^{2}\left[\lambda-\frac{1}{\sqrt{2 \pi}} \exp \left(-\frac{1}{2} \lambda^{2}\right)-\lambda \Phi(\lambda)\right] .
$$

Plugging (39) and (40) into (38), we obtain

$$
\operatorname{Cov}_{t-1}^{Q}\left(\frac{\eta_{t}}{\sigma_{t}}, \sigma_{t+1}^{2}\right)=-2 \sigma_{t}^{2}\left\{\lambda \alpha_{2}+\left[\frac{1}{\sqrt{2 \pi}} \exp \left(-\frac{1}{2} \lambda^{2}\right)+\lambda \Phi(\lambda)\right]\left(\alpha_{1}-\alpha_{2}\right)\right\} .
$$

Noting that $\operatorname{Cov}^{Q}\left(\eta_{t} / \sigma_{t}, \sigma_{t+1}^{2}\right)=\mathrm{E}^{Q}\left[\operatorname{Cov}_{t-1}^{Q}\left(\eta_{t} / \sigma_{t}, \sigma_{t+1}^{2}\right)\right]$, the result is obtained. Q.E.D. 


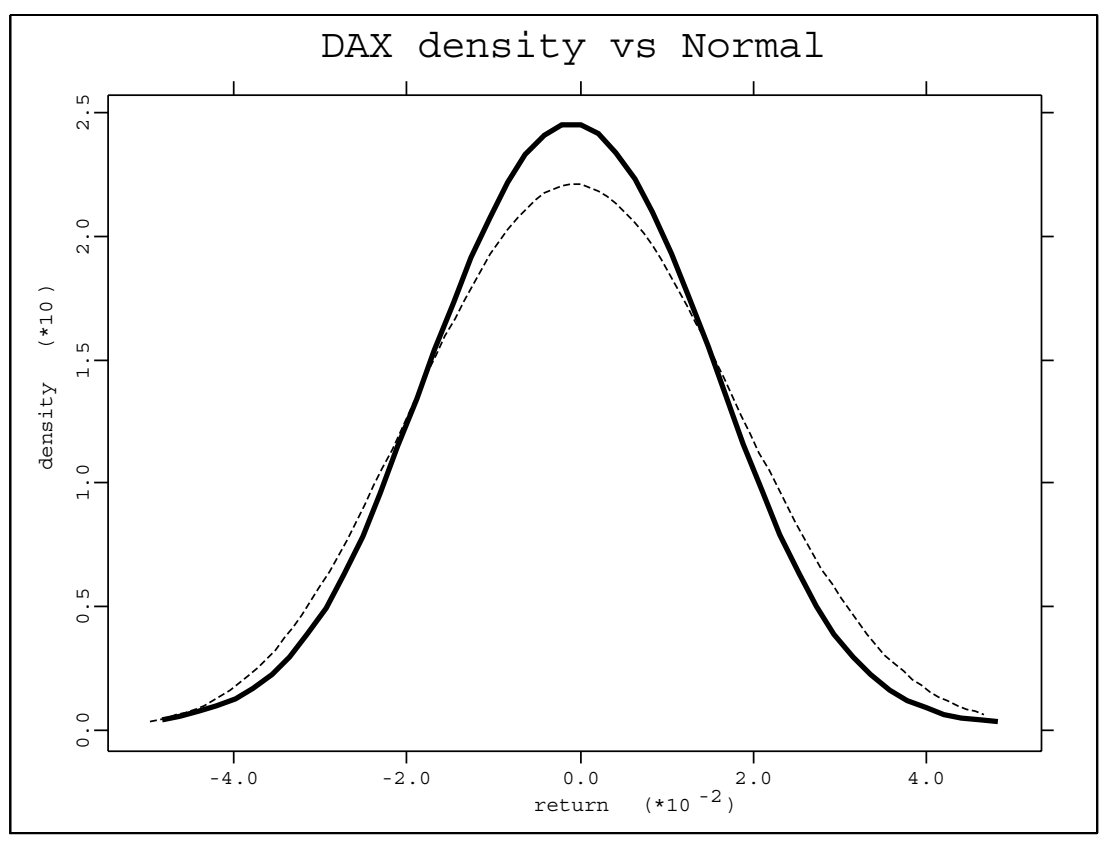

Figure 1: Kernel estimate of the DAX return distribution (solid line) versus a Kernel estimate of a normal distribution (dashed line) with the same mean and variance. We used a bandwidth of 0.03 and a quartic kernel. The boundary regions are skipped in the figure.

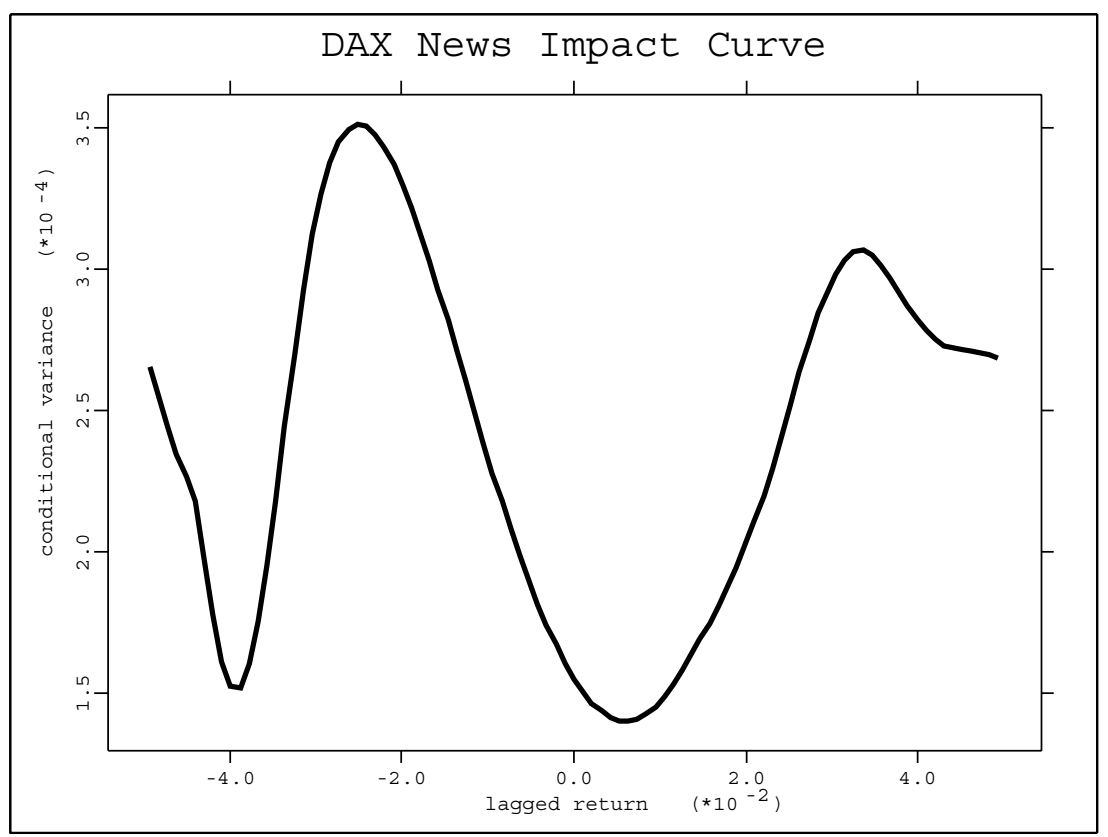

Figure 2: Local linear estimate of the news impact curve for the DAX. The model is $y_{t}=$ $\sigma\left(y_{t-1}\right) \xi_{t}$. Shown is the estimate of the function $\sigma^{2}\left(y_{t-1}\right)$ for a bandwidth choice of 0.03 . The boundary regions are skipped in the figure. 


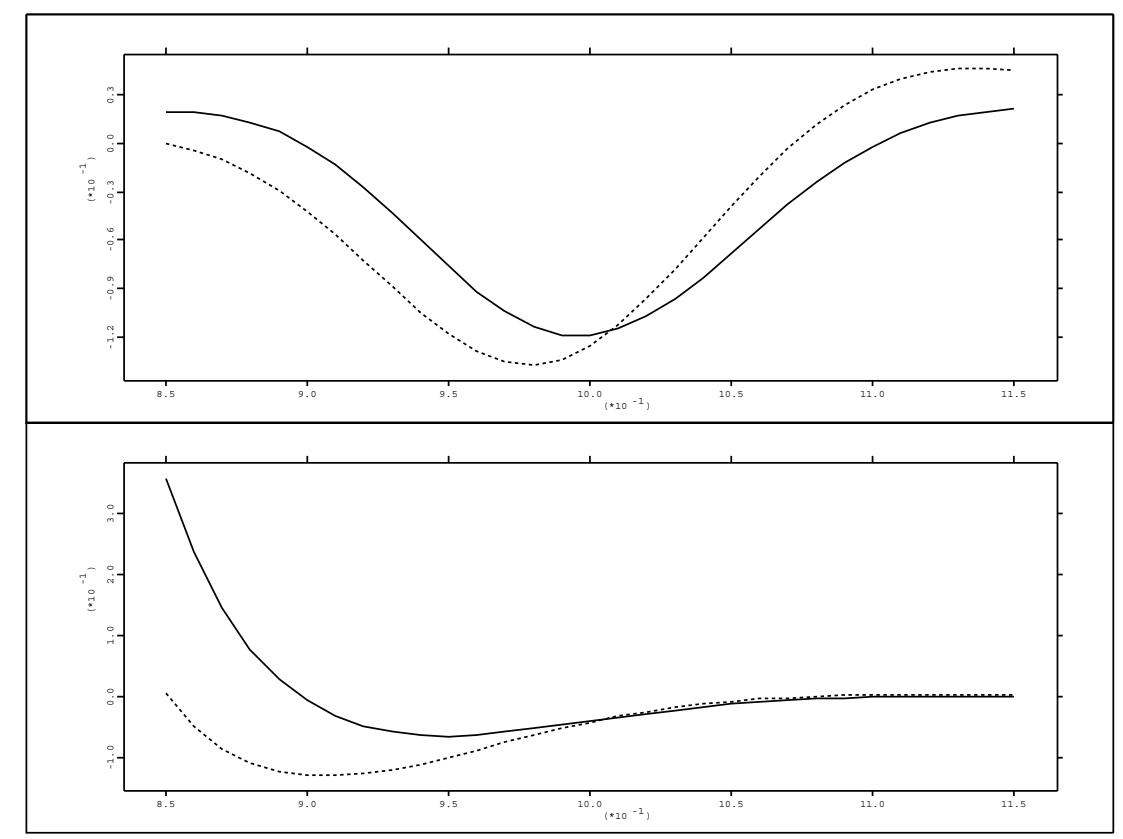

Figure 3: The plot above shows the price difference of simulated GARCH (solid line) and TGARCH (dashed line) option prices to Black/Scholes prices as a function of moneyness. The plot below shows the corresponding differences divided by the Black/Scholes prices. Parameters: Type 1 and leverage effect case (see text).

\begin{tabular}{|c|c|ccc|ccc|}
\hline \hline money & BS & GARCH & $\%$ diff & std.dev. & TGARCH & $\%$ diff & std.dev. \\
\hline 0.85 & 0.0546 & 0.0742 & 35.9470 & 1.6970 & 0.0550 & 0.7460 & 1.3589 \\
0.90 & 0.3265 & 0.3247 & -0.5502 & 0.5633 & 0.2847 & -12.7792 & 0.4985 \\
0.95 & 1.2095 & 1.1333 & -6.3020 & 0.2614 & 1.0911 & -9.7856 & 0.2452 \\
1.00 & 3.0894 & 2.9705 & -3.8498 & 0.1320 & 2.9640 & -4.0606 & 0.1251 \\
1.05 & 5.9756 & 5.9076 & -1.1379 & 0.0568 & 5.9367 & -0.6514 & 0.0523 \\
1.10 & 9.4798 & 9.4780 & -0.0197 & 0.0246 & 9.5128 & 0.3475 & 0.0219 \\
1.15 & 13.1454 & 13.1668 & 0.1623 & 0.0120 & 13.1910 & 0.3466 & 0.0101 \\
\hline \hline
\end{tabular}

Table 5: Monte Carlo Simulation results for the leverage effect case and Type 1. money refers to the moneyness of the call option, BS is the analytic Black/Scholes price using the unconditional volatility of the simulated GARCH process, GARCH and TGARCH are the simulated option prices of 400,000 Monte Carlo replications, \% diff is the percentage difference to the Black/Scholes price, and std.dev. is the standard deviation of \% diff for the simulations. 


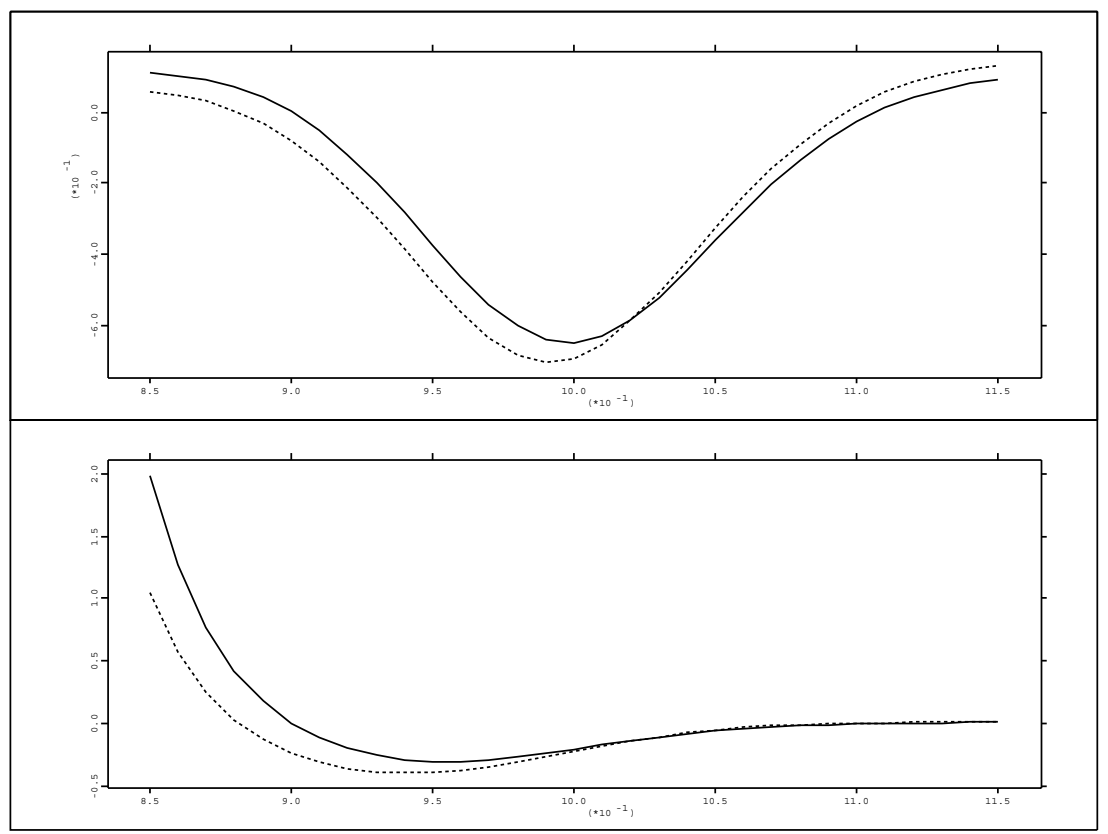

Figure 4: Type 2 and leverage effect case. Notes as in Figure 3.

\begin{tabular}{|c|c|ccc|ccc|}
\hline \hline money & BS & GARCH & \% diff & std.dev. & TGARCH & \% diff & std.dev. \\
\hline 0.85 & 0.0546 & 0.1633 & 199.0678 & 5.8470 & 0.1117 & 104.6195 & 4.4327 \\
0.90 & 0.3265 & 0.3280 & 0.4886 & 1.1363 & 0.2482 & -23.9643 & 0.8906 \\
0.95 & 1.2095 & 0.8375 & -30.7589 & 0.3697 & 0.7340 & -39.3159 & 0.3048 \\
1.00 & 3.0894 & 2.4414 & -20.9751 & 0.1666 & 2.3986 & -22.3622 & 0.1415 \\
1.05 & 5.9756 & 5.6148 & -6.0383 & 0.0769 & 5.6513 & -5.4275 & 0.0629 \\
1.10 & 9.4798 & 9.4512 & -0.3019 & 0.0420 & 9.4990 & 0.2019 & 0.0333 \\
1.15 & 13.1454 & 13.2368 & 0.6952 & 0.0273 & 13.2758 & 0.9915 & 0.0213 \\
\hline \hline
\end{tabular}

Table 6: Simulation results for the leverage effect case and Type 2. Notes as in Table 5. 


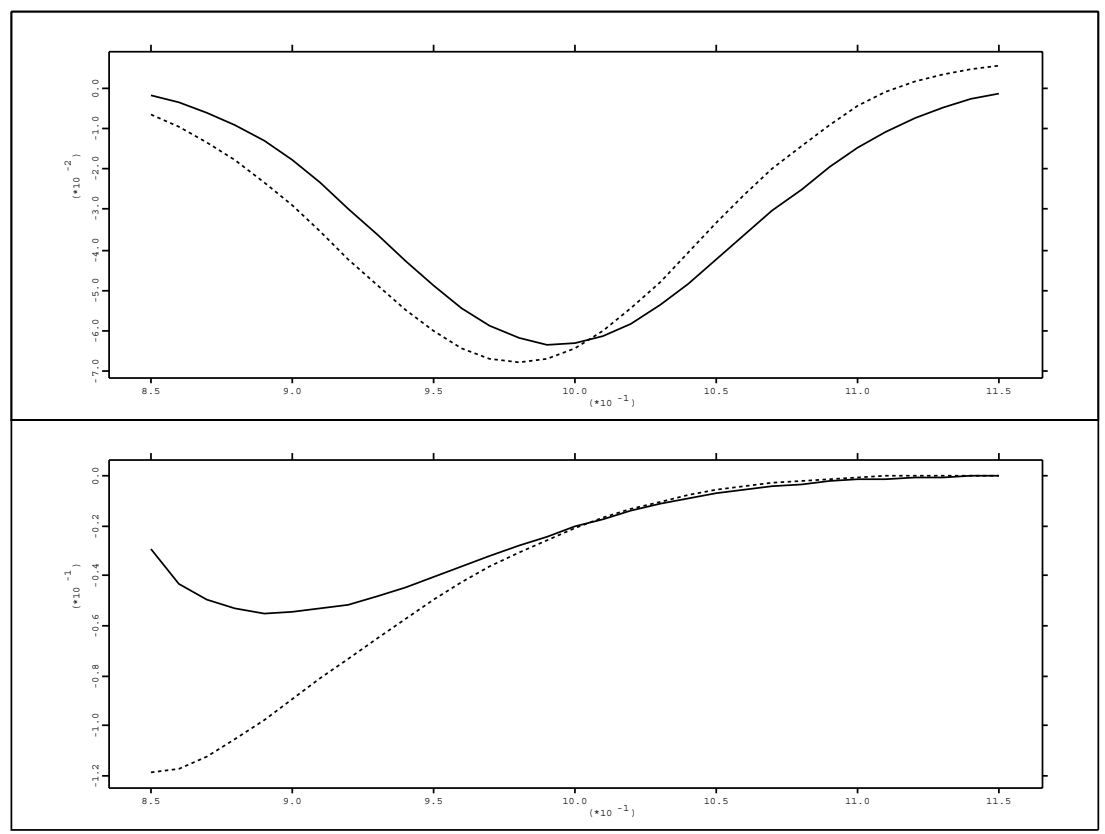

Figure 5: Type 3 and leverage effect case. Notes as in Figure 3.

\begin{tabular}{|c|c|ccc|ccc|}
\hline \hline money & BS & GARCH & $\%$ diff & std.dev. & TGARCH & \% diff & std.dev. \\
\hline 0.8500 & 0.0546 & 0.0530 & -2.8987 & 1.2090 & 0.0481 & -11.8976 & 1.1254 \\
0.9000 & 0.3265 & 0.3087 & -5.4393 & 0.4962 & 0.2974 & -8.8861 & 0.4795 \\
0.9500 & 1.2095 & 1.1608 & -4.0265 & 0.2494 & 1.1494 & -4.9704 & 0.2452 \\
1.0000 & 3.0894 & 3.0263 & -2.0417 & 0.1281 & 3.0252 & -2.0773 & 0.1263 \\
1.0500 & 5.9756 & 5.9332 & -0.7097 & 0.0546 & 5.9422 & -0.5593 & 0.0534 \\
1.1000 & 9.4798 & 9.4649 & -0.1571 & 0.0227 & 9.4753 & -0.0475 & 0.0220 \\
1.1500 & 13.1454 & 13.1442 & -0.0093 & 0.0102 & 13.1510 & 0.0421 & 0.0097 \\
\hline \hline
\end{tabular}

Table 7: Simulation results for the leverage effect case and Type 3. Notes as in Table 5. 


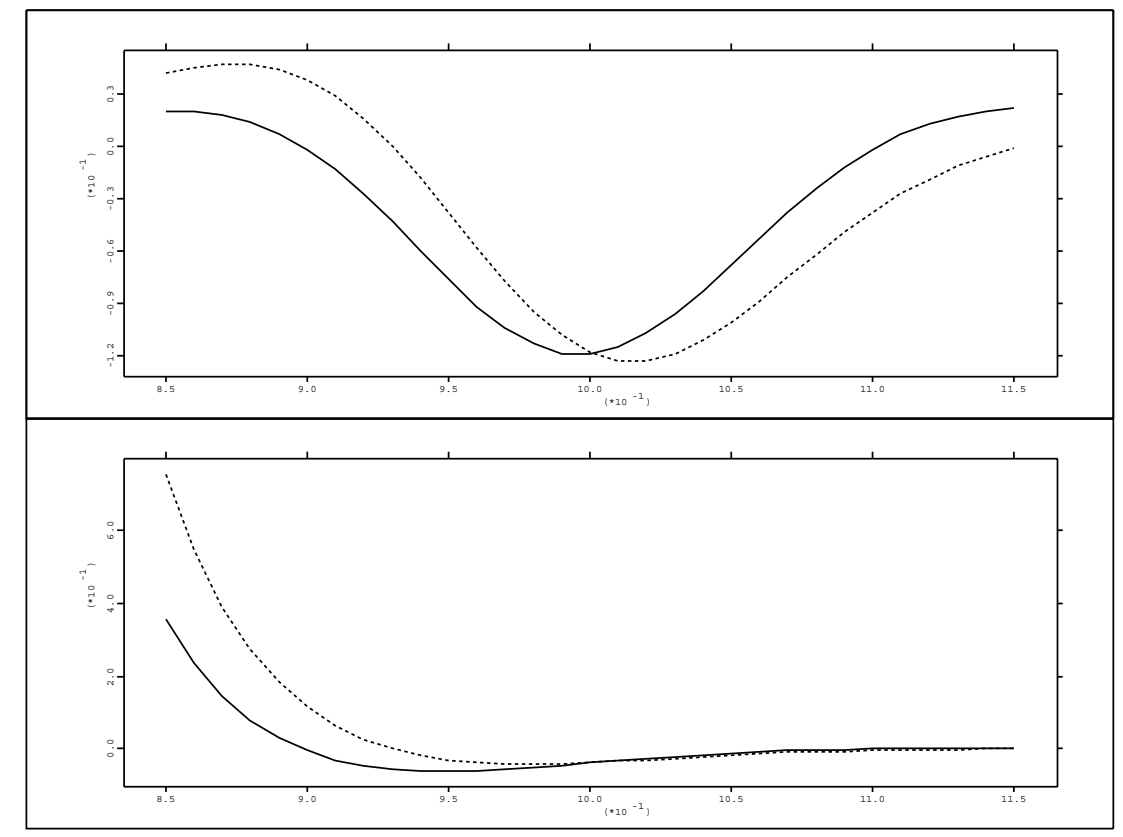

Figure 6: Type 1 and reverted leverage effect case.

Notes as in Figure 3.

\begin{tabular}{|c|c|ccc|ccc|}
\hline \hline money & BS & GARCH & $\%$ diff & std.dev. & TGARCH & \% diff & std.dev. \\
\hline 0.85 & 0.0546 & 0.0742 & 35.9470 & 1.6970 & 0.0960 & 75.7687 & 2.0688 \\
0.90 & 0.3265 & 0.3247 & -0.5502 & 0.5633 & 0.3643 & 11.6064 & 0.6311 \\
0.95 & 1.2095 & 1.1333 & -6.3020 & 0.2614 & 1.1713 & -3.1527 & 0.2782 \\
1.00 & 3.0894 & 2.9705 & -3.8498 & 0.1320 & 2.9718 & -3.8058 & 0.1390 \\
1.05 & 5.9756 & 5.9076 & -1.1379 & 0.0568 & 5.8745 & -1.6922 & 0.0613 \\
1.10 & 9.4798 & 9.4780 & -0.0197 & 0.0246 & 9.4419 & -0.4000 & 0.0276 \\
1.15 & 13.1454 & 13.1668 & 0.1623 & 0.0120 & 13.1437 & -0.0128 & 0.0142 \\
\hline \hline
\end{tabular}

Table 8: Simulation results for the reverted leverage effect case and Type 1 . Notes as in Table 5. 


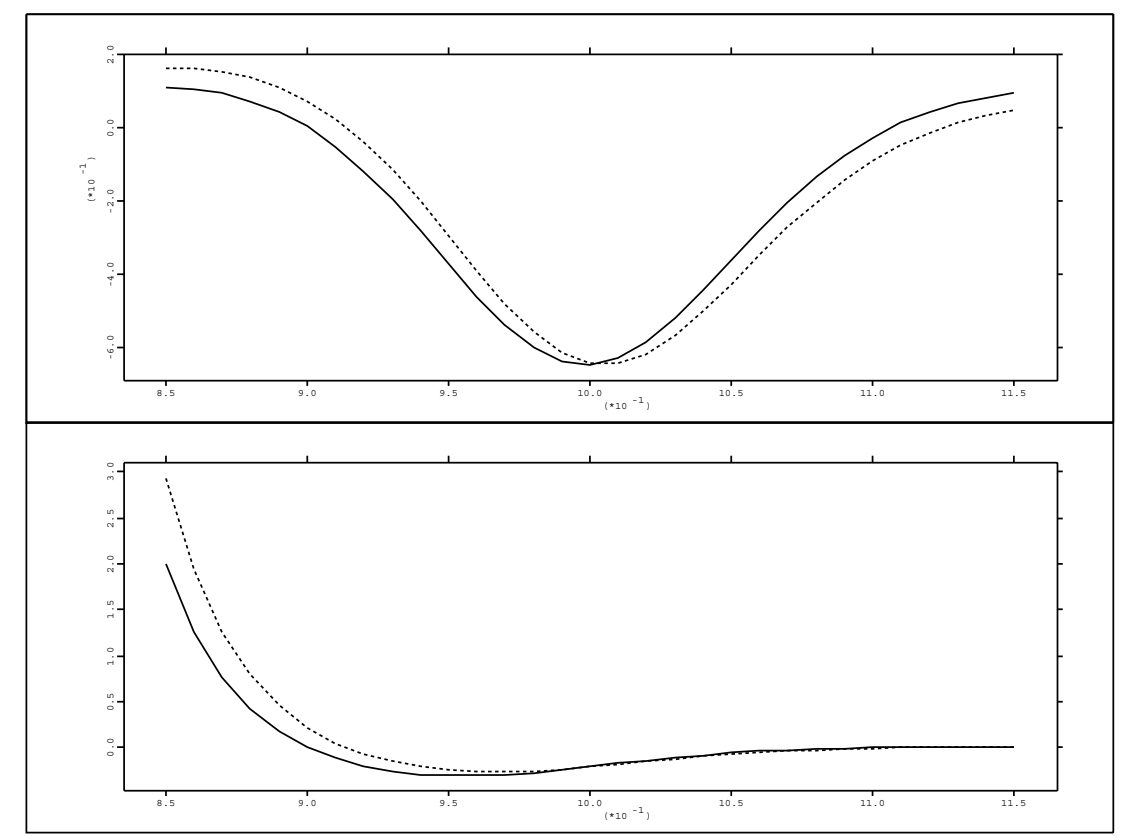

Figure 7: Type 2 and reverted leverage effect case.

Notes as in Figure 3.

\begin{tabular}{|c|c|ccc|ccc|}
\hline \hline money & BS & GARCH & \% diff & std.dev. & TGARCH & \% diff & std.dev. \\
\hline 0.85 & 0.0546 & 0.1633 & 199.0678 & 5.8470 & 0.2149 & 293.7038 & 7.8836 \\
0.90 & 0.3265 & 0.3280 & 0.4886 & 1.1363 & 0.3987 & 22.1405 & 1.4693 \\
0.95 & 1.2095 & 0.8375 & -30.7589 & 0.3697 & 0.9129 & -24.5184 & 0.4540 \\
1.00 & 3.0894 & 2.4414 & -20.9751 & 0.1666 & 2.4467 & -20.8043 & 0.1984 \\
1.05 & 5.9756 & 5.6148 & -6.0383 & 0.0769 & 5.5485 & -7.1476 & 0.0954 \\
1.10 & 9.4798 & 9.4512 & -0.3019 & 0.0420 & 9.3882 & -0.9664 & 0.0544 \\
1.15 & 13.1454 & 13.2368 & 0.6952 & 0.0273 & 13.1915 & 0.3509 & 0.0367 \\
\hline \hline
\end{tabular}

Table 9: Simulation results for the reverted leverage effect case and Type 2. Notes as in Table 5. 


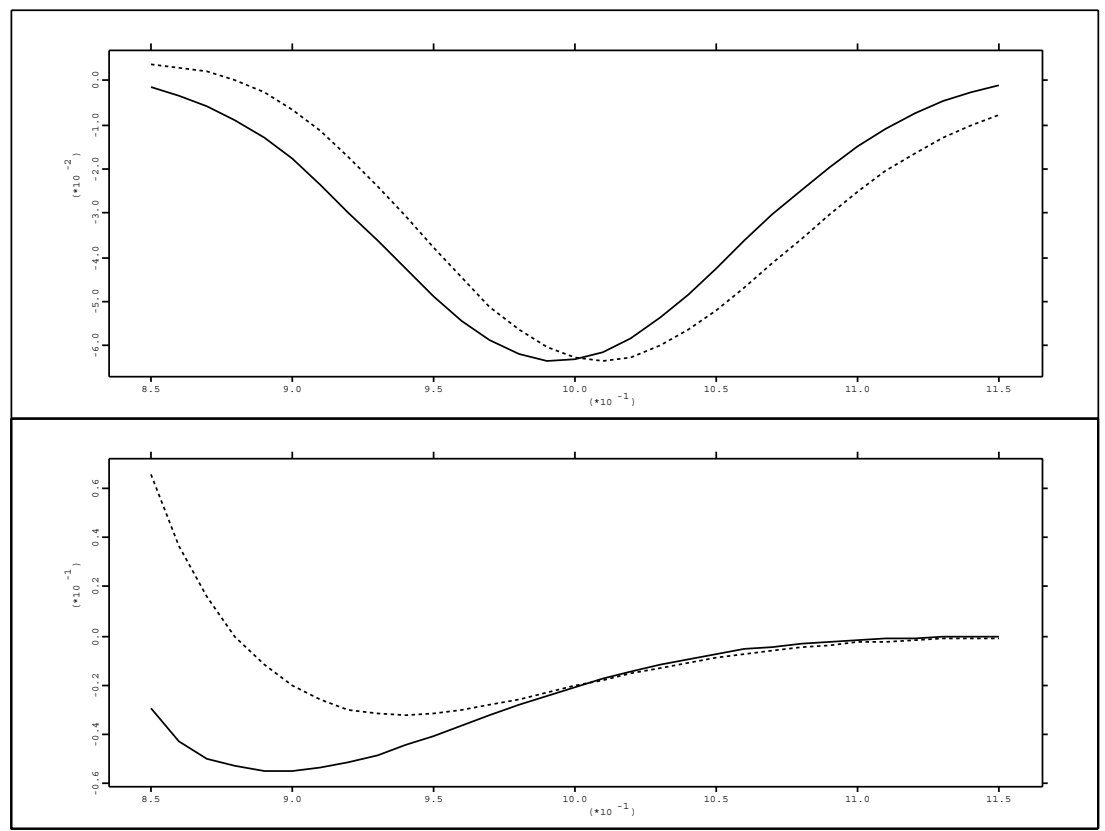

Figure 8: Type 3 and reverted leverage effect case. Notes as in Figure 3.

\begin{tabular}{|c|c|ccc|ccc|}
\hline \hline money & BS & GARCH & \% diff & std.dev. & TGARCH & \% diff & std.dev. \\
\hline 0.85 & 0.0546 & 0.0530 & -2.8987 & 1.2090 & 0.0582 & 6.6867 & 1.2973 \\
0.90 & 0.3265 & 0.3087 & -5.4393 & 0.4962 & 0.3200 & -1.9816 & 0.5135 \\
0.95 & 1.2095 & 1.1608 & -4.0265 & 0.2494 & 1.1718 & -3.1141 & 0.2537 \\
1.00 & 3.0894 & 3.0263 & -2.0417 & 0.1281 & 3.0269 & -2.0246 & 0.1300 \\
1.05 & 5.9756 & 5.9332 & -0.7097 & 0.0546 & 5.9238 & -0.8670 & 0.0559 \\
1.10 & 9.4798 & 9.4649 & -0.1571 & 0.0227 & 9.4545 & -0.2673 & 0.0235 \\
1.15 & 13.1454 & 13.1442 & -0.0093 & 0.0102 & 13.1376 & -0.0591 & 0.0108 \\
\hline \hline
\end{tabular}

Table 10: Simulation results for the reverted leverage effect case and Type 3. Notes as in Table 5. 